\title{
ON THE MEASURE OF THE ONE-SKELETON OF THE SUM OF CONVEX COMPACT SETS
}

\author{
LEONI DALLA
}

(Received 17 June 1985; revised 17 January 1986)

Communicated by $\mathrm{H}$. Lausch

\begin{abstract}
For any two compact convex sets in a Euclidean space, the relation between the volume of the sum of the two sets and the volume of each of them is given by the Brün-Minkowski inequality. In this note we prove an analogous relation for the one-dimensional Hausdorff measure of the one-skeleton of the above sets. Also, some counterexamples are given which show that the above results are the best possible in some special cases.
\end{abstract}

1980 Mathematics subject classification (Amer. Math. Soc.): 52 A 20.

\section{Introduction}

When $K$ is a convex compact subset of a Euclidean space $E^{d}$, then for $\nu=0,1, \ldots, d$, the $\nu$-skeleton skel $_{\nu} K$ of $K$ consists of those points of $K$ which are not centres of $(\nu+1)$-dimensional balls contained in $K$.

It is well known (see Larman and Rogers [4]) that the $\nu$-skeleton of a compact convex set in $E^{d}$ is a measurable set with respect to the $\nu$-dimensional Hausdorff measure, denoted by $\mathscr{H}^{\nu}(\cdot)$. We define $n_{v}(K)=\mathscr{H}^{\nu}\left(\operatorname{skel}_{\nu} K\right)$. If $K_{0}$ and $K_{1}$ are compact convex subsets of $E^{d}$, then it is known that the $d$ th root of $n_{d}(\cdot)$ is a concave function, i.e. for any $0 \leqslant t \leqslant 1$, if $K_{t}=(1-t) K_{0}+t K_{1}$, then

$$
\left(n_{d}\left(K_{t}\right)\right)^{1 / d} \geqslant(1-t)\left(n_{d}\left(K_{0}\right)\right)^{1 / d}+t\left(n_{d}\left(K_{1}\right)\right)^{1 / d}
$$

for any $K_{0}, K_{1}$. This inequality is known as the Brünn-Minkowski inequality.

(C) 1987 Australian Mathematical Society 0263-6115/87 \$A2.00+0.00 
In this note we prove that $n_{1}(\cdot)$ is a concave function, i.e.

$$
n_{1}\left(K_{t}\right) \geqslant(1-t) n_{1}\left(K_{0}\right)+t n_{1}\left(K_{1}\right)
$$

for any compact convex sets $K_{0}, K_{1}$ of $E^{d}$.

In the course of the proof of the above property, we establish an inequality between $\mathscr{H}^{s}\left(\right.$ ext $\left.K_{t}\right)$ and $\mathscr{H}^{s}$ (ext $\left.K_{i}\right), i=0,1$, for any $s \geqslant 0$, where ext $K$ denotes the set of extreme points of $K$.

We also prove, constructing appropriate counterexamples in $E^{3}$, that the two inequalities cannot be reversed.

\section{The results}

We quote first a lemma which is to be used in the proofs that follow.

LEMMA 2.1. Let $K_{1}, K_{2}$ be convex, compact subsets of $E^{d}$ and let $K=\lambda K_{1}+$ $\mu K_{2}, \lambda, \mu \geqslant 0$, be a Minkowski linear combination of $K_{1}$ and $K_{2}$, where $\lambda, \mu$ are fixed but arbitrary. Then the following hold.

(i) For any point e belonging to the set ext $K$ of the extreme points of $K$, there exist uniquely defined points $e_{1}, e_{2}$, where $e_{i} \in \operatorname{ext} K_{i}, i=1,2$, such that $e=\lambda e_{1}$ $+\mu e_{2}$.

(ii) For any point $e_{1} \in \operatorname{ext} K_{1}$, there exists a point $e_{2} \in$ ext $K_{2}$ such that $\left(\lambda e_{1}+\mu e_{2}\right) \in \operatorname{ext} K$. A similar property holds for the extreme points of $K_{2}$.

Proof. If either $\lambda=0$ or $\mu=0$, the results are obvious. Suppose now that $\lambda$, $\mu \neq 0$. We consider part (i). Let $e \in \operatorname{ext} K$, with $e=\lambda e_{1}+\mu e_{2}$ for some $e_{i} \in K_{i}$, $i=1,2$. If $e_{1} \notin$ ext $K_{1}$, then $e_{1}=\left(x_{1}+y_{1}\right) / 2$ for some $x_{1}, y_{1} \in K_{1}$ with $x_{1} \neq y_{1}$, and so $e=\left(\lambda x_{1}+\mu e_{2}\right) / 2+\left(\lambda y_{1}+\mu e_{2}\right) / 2$. But $\lambda x_{1}+\mu e_{2}$ and $\lambda y_{1}+\mu e_{2}$ are distinct points of $K$, which is a contradiction, as $e \in \operatorname{ext} K$. Hence $e_{1} \in \operatorname{ext} K_{1}$. In a similar way $e_{2} \in$ ext $K_{2}$.

Suppose now that there exist another pair $e_{1}^{\prime}, e_{2}^{\prime}$, where $e_{i}^{\prime} \in \operatorname{ext} K_{i},=1,2$, such that $e=\lambda e_{1}+\mu e_{2}=\lambda e_{1}^{\prime}+\mu e_{2}^{\prime}$. Then $e=\lambda\left(e_{1}+e_{1}^{\prime}\right) / 2+\mu\left(e_{2}+e_{2}^{\prime}\right) / 2$, which implies that $\left(e_{i}+e_{i}^{\prime}\right) / 2 \in \operatorname{ext} K_{i}, i=1,2$. This, in turn, implies that $e_{i}=e_{i}^{\prime}, i=1,2$, which proves part (i).

For part (ii), consider $e_{1} \in \operatorname{ext} K_{1}$. If $u$ is a unit vector, let $K_{u}^{(1)}$ denote the intersection of $K_{1}$ with its support hyperplane with outer normal $u$. If $U=$ $\left(u_{1}, \ldots, u_{k}\right)$ is a $k$-frame of orthogonal unit vectors, then $K_{U}^{(1)}$ is defined recursively by $K_{\left(u_{1}, \ldots, u_{k}\right)}^{(1)}=\left(K_{\left(u_{1}, \ldots, u_{k-1}\right)}\right)_{u_{k^{*}}}$

Now for the point $e_{1}$ there exists a $k$-frame $U=\left(u_{1}, \ldots, u_{k}\right), 1 \leqslant k \leqslant d$, such that $\left\{e_{1}\right\}=K_{U}^{(1)}$. If $K_{U}^{(2)}, K_{U}$ are the corresponding sets for $K_{2}$ and $K$, then $K_{U}=\lambda K_{U}^{(1)}+\mu K_{U}^{(2)}$ (see Eggleston [3, Theorem 38]). Hence $K_{U}=\lambda\left\{e_{1}\right\}+$ $\mu K_{U}^{(2)}$, and from part (i) we have

$$
\operatorname{ext} K_{U} \subseteq \lambda\left\{e_{1}\right\}+\mu \operatorname{ext} K_{U}^{(2)}
$$


Consider $z \in$ ext $K_{U} \subseteq$ ext $K$. Then $z=\lambda e_{1}+\mu e_{2}$ for some $e_{2} \in \operatorname{ext} K_{U}^{(2)} \subseteq$ ext $K_{2}$. Therefore, for $e_{1} \in \operatorname{ext} K_{1}$, there exists $e_{2} \in \operatorname{ext} K_{2}$ with $\lambda e_{1}+\mu e_{2} \in$ ext $K$. This concludes the proof of (ii).

Now we quote and prove the following propositions

Proposition 2.1. Let $K_{1}, K_{2}$ and $K$ be defined as in Lemma 2.1. Then $\mathscr{H}^{s}(\operatorname{ext} K) \geqslant \max \left\{\lambda^{s} \mathscr{H}^{s}\left(\operatorname{ext} K_{1}\right), \mu^{s} \mathscr{H}^{s}\left(\right.\right.$ ext $\left.\left.K_{2}\right)\right\}$ for any non-negative number $s$.

Proof. As $\mathscr{H}^{s}\left(\operatorname{ext}\left(\lambda K_{1}\right)\right)=\lambda^{s} \mathscr{H}^{s}\left(\operatorname{ext} K_{1}\right)$, to prove the inequality, it is sufficient to prove it for $\lambda=\mu=1$. If $e \in \operatorname{ext} K$, then the cap-neighbourhoods of $e$ form a basis for the neighborhoods of $e$ (see G. Choquet [2], page 107). Therefore $\mathscr{H}^{s}(\operatorname{ext} K)=\sup _{\varepsilon}>0 \inf \left\{\sum_{n=1}^{\infty} d^{s}\left(C_{n}\right): C_{n}, \quad n=1,2, \ldots\right.$, are caps; ext $K \subseteq$ $\left.\bigcup_{n=1}^{\infty} C_{n} ; d\left(C_{n}\right)<\varepsilon\right\}$.

Let $C_{n}, n=1,2, \ldots$, be a sequence of caps of $K$ covering ext $K$, where $C_{n}=\left\{x \in K: a_{n}-t_{n} \leqslant x \cdot u_{n} \leqslant a_{n}\right\}$, where $a_{n}=\sup _{x \in K} x \cdot u_{n}$, and where $x \cdot$ $u_{n}$ denotes the inner-product of $x$ with a unit vector $u_{n}$. We define $C_{n}^{(i)}=\{x \in$ $\left.K_{i}: b_{n}^{(i)}-t_{n} \leqslant x \cdot u_{n} \leqslant b_{n}^{(i)}\right\}, i=1,2$, where $b_{n}^{(i)}=\sup _{x \in K_{i}} x \cdot u_{n}, i=1,2$. Then $a_{n}=b_{n}^{(1)}+b_{n}^{(2)}$. We shall prove that ext $K_{i} \subseteq \bigcup_{n=1}^{\infty} C_{n}^{(i)}, i=1,2$. Let $e_{1} \in \operatorname{ext} K_{1}$. Then by part (ii) of Lemma 2.1 there exists $e_{2} \in \operatorname{ext} K_{2}$ such that $\left(e_{1}+e_{2}\right) \in$ ext $K$. Let $e_{1}+e_{2} \in C_{n}$ for some $n \in N$. Then $e_{i} \in C_{n}^{(i)}, i=1,2$. For, if not, then $e_{1} \notin C_{n}^{(1)}$, say. Then $e_{1} \cdot u_{n}<b_{n}^{(1)}-t_{n}$, so $\left(e_{1}+e_{2}\right) \cdot u_{n}<\left(b_{n}^{(1)}-t_{n}\right)+b_{n}^{(2)}$ $=a_{n}-t_{n}$. This is impossible since $e_{1}+e_{2} \in C_{n}$. Hence, for any $e_{1} \in \operatorname{ext} K_{1}$, there exists a cap $C_{n}^{(1)}$ such that $e_{1} \in C_{n}^{(1)}$, and so ext $K_{1} \subseteq \bigcup_{n=1}^{\infty} C_{n}$. We also have $d\left(C_{n}^{(i)}\right) \leqslant d\left(C_{n}\right), i=1,2, n \in \mathbb{N}$. Indeed, for $\beta_{2} \in K_{2}$ with $\beta_{2} \cdot u_{n}=b_{n}^{(2)}$, we have $C_{n}^{(1)}+\beta_{2} \subseteq C_{n}$, and so $d\left(C_{n}^{(1)}\right)=d\left(C_{n}^{(1)}+\beta_{2}\right) \leqslant d\left(C_{n}\right)$. Then $\inf \left\{\sum_{n=1}^{\infty} d\left(S_{n}\right): \operatorname{ext} K_{i} \subseteq \bigcup_{n=1}^{\infty} S_{n}, d\left(S_{n}\right) \leqslant \varepsilon\right\} \leqslant \inf \left\{\sum_{n=1}^{\infty} d^{s}\left(C_{n}\right): \operatorname{ext} K \subseteq\right.$ $\cup_{n-1}^{\infty} C_{n}, d\left(C_{n}\right)<\varepsilon, C_{n}$ cap, $\left.n \in \mathbb{N}\right\}$ for any $\varepsilon>0$. Therefore $\mathscr{H}^{s}\left(\operatorname{ext} K_{i}\right) \leqslant$ $\mathscr{H}^{s}($ ext $K), i=1,2$. This concludes the proof of the proposition.

We note that in general no kind of reverse inequality holds.

More precisely, we show, by constructing a counterexample, that there does not exist a positive constant $M$ such that the inequality

$$
\mathscr{H}^{1}(\operatorname{ext} K) \leqslant M\left(\max \left\{\mathscr{H}^{1}\left(\operatorname{ext} K_{1}\right), \mathscr{H}^{1}\left(\operatorname{ext} K_{2}\right)\right\}\right)
$$

holds for any compact convex sets $K_{1}, K_{2}$ in $E^{3}$. Indeed, take $K_{1}=\{(x, 0, z) \in$ $\left.\mathbb{R}^{3}: x \geqslant 0, z \geqslant 0,\left(x^{2}+z^{2}\right)^{1 / 2} \leqslant 1\right\}$ and $K_{2}=\left\{(0, y, z) \in \mathbb{R}^{3}: y \geqslant 0, z \geqslant 0\right.$, $\left.\left(y^{2}+z^{2}\right)^{1 / 2} \leqslant 1\right\}$. Then $\mathscr{H}^{1}\left(\operatorname{ext} K_{1}\right)=\mathscr{H}^{1}\left(\operatorname{ext} K_{2}\right)=\pi / 2<+\infty$. The sum of $K_{1}$ and $K_{2}$ is the set $K=\left\{(x, y, z) \in \mathbb{R}^{3}: 0 \leqslant x \leqslant 1,0 \leqslant y \leqslant 1,0 \leqslant z \leqslant\right.$ $\left.\left(1-x^{2}\right)^{1 / 2}+\left(1-y^{2}\right)^{1 / 2}\right\}$, and ext $K=\left\{(x, y, z) \in \mathbb{R}^{3}: 0 \leqslant x \leqslant 1,0 \leqslant y \leqslant 1\right.$, $\left.z=\left(1-x^{2}\right)^{1 / 2}+\left(1-y^{2}\right)^{1 / 2}\right\} \cup\{(0,0,0)\} \cup\{(1,0,0)\} \cup\{(0,1,0)\}$. Therefore $\mathscr{H}^{2}(\operatorname{ext} K)>0$. But then $\mathscr{H}^{1}(\operatorname{ext} K)=+\infty$, and in fact ext $K$ is not $\sigma$-finite with respect to $\mathscr{H}^{1}$. 
Proposition 2.2. Let $K_{1}, K_{2}$ and $K$ be as in Lemma 2.1. Then $\mathscr{H}^{1}\left(\operatorname{skel}_{1} K\right) \geqslant$ $\lambda \mathscr{H}^{1}\left(\right.$ skel $\left._{1} K_{1}\right)+\mu \mathscr{H}^{1}\left(\right.$ skel $\left._{1} K_{2}\right)$.

Proof. As in Proposition 2.1, it is sufficient to prove the inequality for $\lambda=\mu=1$. Then $K=K_{1}+K_{2}$. If $\mathscr{H}^{1}\left(\right.$ skel $\left._{1} K\right)=+\infty$, we have nothing to prove.

Assume now that $\mathscr{H}^{1}$ (skel ${ }_{1} K$ ) $<\infty$. It is known, (see Burton [4, Theorems 1 and 3]), that skel $K$ is the union of ext $K$ with countably many exposed edges $F_{n}$ $(n=1,2, \ldots)$, and that $\mathscr{H}^{1}(\operatorname{ext} K)=0$. Hence $\mathscr{H}^{1}\left(\operatorname{skel}_{1} K\right)=\sum_{n=1}^{\infty} \mathscr{H}^{1}\left(F_{n}\right)$ and, by Proposition 2.1, $\mathscr{H}^{1}\left(\operatorname{ext} K_{i}\right)=0, i=1,2$.

Now $F_{n}=K \cap H=K_{1} \cap H_{1}+K_{2} \cap H_{2}$, where $H$ is the support hyperplane of $K$ at $F_{n}$, and where $H_{1}, H_{2}$ are the corresponding suport hyperplanes of $K_{1}$, $K_{2} . A s \operatorname{dim}\left(F_{n}\right)=1$, we conclude that $F_{n}=l_{1}+l_{2}$, where $l_{1}$ and $l_{2}$ are parallel line segments which are edges of $K_{1}$ and $K_{2}$; or $F_{n}=l_{1}+\left\{e_{2}\right\}$, where $l_{1}$ is an edge of $K_{1}$ and $e_{2}$ an exposed point of $K_{2}$; or $F_{n}=\left\{e_{1}\right\}+l_{2}$, where $l_{2}$ is an edge of $K_{2}$ and $e_{1}$ an exposed point of $K_{1}$. The above expression is uniquely determined. Suppose, for example, that $F_{n}=l_{1}+l_{2}=l_{1}^{\prime}+\left\{e_{2}^{\prime}\right\}$, where $l_{1}, l_{1}^{\prime}$ are edges of $K_{1}$, where $l_{2}$ is an edge of $K_{2}$, and where $e_{2}^{\prime}$ is an exposed point of $K_{2}$. Then $F_{n}=\left(l_{1}+l_{1}^{\prime}\right) / 2+\left(l_{2}+\left\{e_{2}^{\prime}\right\}\right) / 2$, which implies that $\frac{1}{2}\left(l_{1}+l_{1}^{\prime}\right)$ is an edge of $K_{1}$; but since $\left(l_{1}+l_{1}^{\prime}\right) / 2 \subset \operatorname{conv}\left(l_{1}, l_{1}^{\prime}\right)$, we have $l_{1}=l_{1}^{\prime}$. Therefore, $F_{n}=l_{1}+$ $l_{2}=l_{1}+\left\{e_{2}^{\prime}\right\}$, and hence $\left\{e_{2}^{\prime}\right\}=l_{2}$. Similar arguments apply to the other possible expressions for $F_{n}$.

Let $l_{1}$ be an edge of $K_{1}$. We denote by $\operatorname{pr}(\cdot)$ the projection onto $E^{d-1}$ which maps in the direction of $l_{1}$. Then $\operatorname{pr}(K)=\operatorname{pr}\left(K_{1}\right)+\operatorname{pr}\left(K_{2}\right)$, and $\operatorname{pr}\left(l_{1}\right)$ is an extreme point of $\operatorname{pr}\left(K_{1}\right)$. Then, from Proposition 2.1, there exists an extreme point, say, $e_{2}$, of $\operatorname{pr}\left(K_{2}\right)$ such that $\operatorname{pr}\left(l_{1}\right)+e_{2}=e$, where $e \in \operatorname{ext} \operatorname{pr}(K)$. Then $\mathrm{pr}^{-1}(e) \cap K=l_{1}+\mathrm{pr}^{-1}\left(e_{2}\right) \cap K_{2}$. From the last relation and from the fact that $e$ is an extreme point of $\operatorname{pr}(K)$, we conclude that $\operatorname{pr}^{-1}(e) \cap K$ must be an edge of $K$, and that $\mathrm{pr}^{-1}\left(e_{2}\right) \cap K_{2}$ must be an extreme point or an edge of $K_{2}$. Hence, for each edge $l_{1}$ of $K_{1}$, there exists an extreme point $e_{2}$ or an edge $l_{2}$ of $K_{2}$ such that either $l_{1}+l_{2}$ or $l_{1}+e_{2}$ is an edge of $K$. From the above we conclude that a given edge $l_{i}$ of $K_{i}$ could give rise to more than one edge of $K$. So the edges of $K_{1}$ and $K_{2}$ are countable, and $\operatorname{skel}_{1} K_{i}=\bigcup_{n=1}^{\infty}\left(l_{n}^{i} \cup \operatorname{ext} K_{i}\right), i=1,2$. Hence

$$
\mathscr{H}^{1}\left(\operatorname{skel}_{1} K\right) \geqslant \sum_{n=1}^{\infty} \mathscr{H}^{1}\left(l_{n}^{1}\right)+\sum_{n=1}^{\infty} \mathscr{H}^{1}\left(l_{n}^{2}\right)=\mathscr{H}^{1}\left(\operatorname{skel}_{1} K_{1}\right)+\mathscr{H}^{1}\left(\operatorname{skel}_{1} K_{2}\right),
$$

as $\mathscr{H}^{1}\left(\right.$ ext $\left.K_{i}\right)=0, i=1,2$. This concluces the proof of the proposition.

An immediate consequence of Proposition 2.2 is the following corollary, whose proof is obvious.

COROLlaRY 2.1. The function $n_{1}(\cdot)$ is a concave function. 
In the same way as in Proposition 2.1, we assert that there does not exist a positive number $M$ such that $\mathscr{H}^{1}\left(\operatorname{skel}_{1} K\right) \leqslant M\left[\lambda \mathscr{H}^{1}\left(\operatorname{skel}_{1} K_{1}\right)+\right.$ $\left.\mu \mathscr{H}^{1}\left(\operatorname{skel}_{1} K_{2}\right)\right]$ for any compact convex sets $K_{1}, K_{2}$ in $E^{3}$. To show this, we construct two convex compact sets $A_{1}$ and $A_{2}$ in $E^{3}$ such that $\mathscr{H}^{1}\left(\operatorname{skel}_{1} A_{i}\right)<$ $+\infty, i=1,2$, while $\mathscr{H}^{1}\left(\operatorname{skel}_{1}\left(A_{1}+A_{2}\right)\right)=+\infty$. Let $u_{1}(0,2,0), u_{2}=(0,-2,0)$, $\beta_{0}=(2,2,0), \gamma_{0}=(2,-2,0), \alpha_{0}=(2,0,1)$ and $\delta_{0}=(0,0,1)$. Define $K_{0}$ to be convex hull of these points and let $l=\left[\alpha_{0}, \delta_{0}\right]$. We consider a plane $H_{1}$ such that $(0,0,0) \in H_{1}^{+}, \quad \alpha_{0} \in H_{1}^{-}$, and $K_{0} \cap H_{1}$ is an isosceles triangle $T_{1}=$ $\operatorname{conv}\left(\alpha_{1}, \beta_{1}, \gamma_{1}\right)$ with $\left|\alpha_{1}-\beta_{1}\right|=\left|\alpha_{1}-\gamma_{1}\right|$, with diameter $\left(T_{1}\right)=2^{-1}$, with $\alpha_{1} \in l$, and with the line segment $\left[\beta_{1}, \gamma_{1}\right]$ parallel to $\left[\beta_{0}, \gamma_{0}\right]$. Define $K_{1}=H_{1}^{+} \cap K_{0}$. We now proceed inductively. Assuming that we have constructed $K_{n}(n \geqslant 1)$, we choose the plane $H_{n+1}$ in such a way that $(0,0,0) \in H_{n+1}^{+}$, that $\alpha_{n} \in H_{n+1}^{-}$, and that $K_{n} \cap H_{n+1}$ is an isosceles triangle $T_{n+1}=\operatorname{conv}\left(\alpha_{n+1}, \beta_{n+1}, \gamma_{n+1}\right)$ with $\mid \alpha_{n+1}$ $-\beta_{n+1}|=| \alpha_{n+1}-\gamma_{n+1} \mid$, with diameter $\left(T_{n+1}\right)=2^{-(n+1)}$, with $\alpha_{n+1} \in l$, and with the line segment $\left[\beta_{n+1}, \gamma_{n+1}\right]$ parallel to $\left[\beta_{0}, \gamma_{0}\right]$. Then $K_{n+1}=H_{n+1}^{+} \cap K_{n}$.

Now let $A_{1}=\lim _{n \rightarrow \infty} K_{n}=\bigcap_{n=0}^{\infty} K_{n}=K_{0} \cap \bigcap_{n=1}^{\infty} H_{n}^{+}$. Then $A_{1}=$ $\operatorname{clconv}\left\{\left\{u_{1}\right\} \cup\left\{u_{2}\right\} \cup\left\{\delta_{0}\right\} \cup \bigcup_{n=0}^{\infty}\left\{\beta_{n}\right\} \cup \cup_{n=0}^{\infty}\left\{\gamma_{n}\right\}\right\}$, and $\operatorname{skel}_{1} A_{1}=\left[u_{1}, u_{2}\right]$ $\cup\left[u_{1}, \delta_{0}\right] \cup\left[u_{2}, \delta_{0}\right] \cup\left[u_{1}, \beta_{0}\right] \cup\left[u_{2}, \gamma_{0}\right] \cup \cup_{n=0}^{\infty}\left[\beta_{n}, \gamma_{n}\right] \cup \cup_{n=0}^{\infty}\left[\beta_{n}, \beta_{n+1}\right] \cup$ $\cup_{n=0}^{\infty}\left[\gamma_{n}, \gamma_{n+1}\right] \cup\left[\delta_{0}, \delta_{1}\right]$, where $\delta_{1}=\lim _{n \rightarrow \infty} \beta_{n}=\lim _{n \rightarrow \infty} \gamma_{n}$. Then $\mathscr{H}^{1}\left(\right.$ skel $\left._{1} A_{1}\right) \leqslant \mathscr{H}^{1}\left(\operatorname{skel}_{1} K_{0}\right)+\sum_{n=1}^{\infty} \mathscr{H}^{1}\left(\left[\beta_{n}, \gamma_{n}\right]\right) \leqslant \mathscr{H}^{1}\left(\right.$ skel $\left._{1} K_{0}\right)+\sum_{n=1}^{\infty} 2^{-n}<$ $+\infty$. On the other hand, we define $A_{2}$ to be the orthogonal parallelogram with vertices $u_{1}, u_{2}, \beta_{0}, \gamma_{0}, u_{1}+\delta_{0}, u_{1}+\beta_{0}, \beta_{0}+\delta_{0}$ and $\gamma_{0}+\delta_{0}$. Obviously $\mathscr{H}^{1}$ (skel $\left.A_{2}\right)<+\infty$. But the sum $A_{1}+A_{2}$ has in its 1 -skeleton countably many edges with length greater than 4 . Hence $\mathscr{H}^{1}\left(\operatorname{skel}_{1}\left(A_{1}+A_{2}\right)\right)=+\infty$. From this the assertion follows.

\section{References}

[1] G. R. Burton, The measure of the s-skeleton of a convex body, Mathematica 26 (1979), 290-301.

[2] G. Choquet, Lectures in Analysis, Vol. II (W. A. Benjamin, New York, Amsterdam, 1969).

[3] H. G. Eggleston, Convexity (Cambridge Univ. Press, 1958).

[4] D. G. Larman and C. A. Rogers, The finite dimensional skeleton of a compact convex set, Bull. London Math. Soc. 5 (1973), 145-153.

\section{Department of Mathematics}

University of Athens

Panepistemiopolis

15781 Athens

Greece 